\title{
Erratum to: Mathematical model of a fluxgate magnetometer
}

Pavel F. Baranov, Vitalia E. Baranova, and Tamara G. Nesterenko

National Research Tomsk Polytechnic University, 634050 Tomsk, Russia

Original article: MATEC Web of Conferences 158, 01006 (2018), https://doi.org/10.1051/matecconf/201815801006

The acknowledgements of the article should be replaced by the following text:

This work was performed in National Research Tomsk Polytechnic University. The research financially supported by the Ministry of Education and Science of the Russian Federation (agreement No.14.578.21.0232, unique identifier RFMEFI57817X232). 